\title{
Sayısal İntegral Metodu ile Singüler Pertürbe Multi-Point Sınır Değer Problemlerinin Sayısal Çözümü
}

\author{
Derya ARSLAN* \\ Bitlis Eren Üniversitesi, Fen Edebiyat Fakültesi, Matematik Bölümü, Bitlis, Türkiye \\ (ORCID: 0000-0001-6138-0607)
}

\begin{abstract}
$\ddot{O} \mathbf{z}$
Bu çalışmada, sağ ya da sol sınır katlarına sahip singüler pertürbe multi-point sınır değer probleminin çözümü için düzgün şebeke üzerinde sayısal integral metodu sunulur. Bu metodun yanında yamuk metodu, sonlu fark metodu ve Thomas algoritmasından yararlanılır. Önerilen yöntemin kararlılığı ve yakınsaklığı analiz edilir. Son olarak da, yöntemin doğruluğunu ve verimliliğini gösteren örnek uygulamalar yapılır.
\end{abstract}

Anahtar kelimeler: Singüler Pertürbe Multi-point Sınır Değer Problemi, Sayısal İntegral Metodu, Yamuk Metodu, Sonlu Fark Metodu, Sağ (Sol) Sınır Katı, Düzgün Şebeke.

\section{A Numerical Solution for Singularly Perturbed Multi-Point Boundary Value Problems with the Numerical Integration Method}

\begin{abstract}
In this study, the numerical integral method on a uniform mesh presented to solve the singularly perturbed multipoint boundary value problem with the right or left boundary layer. Besides this method, trapezoid method, finite difference method and Thomas algorithm are used. The stability and convergence of the proposed method is analyzed. Finally, sample applications are performed to demonstrate the accuracy and efficiency of the method.
\end{abstract}

Keywords: Singulary Perturbed Multi-Point Boundary Value Problem, Numerical Integration Method, Trapezoidal Method, Finite Difference Method, Right (Left) Boundary Layer, Uniform Mesh.

\section{Giriş}

Singüler pertürbe denklemin en yüksek mertebeli türevinin katsayısı, sıfırdan büyük, birden çok küçük olan bir $\varepsilon$ parametresidir. Bu parametreden dolayı tanım bölgesinde sınır katları (yani ince geçiş katları) oluşmaktadır. Buralarda çözümün davranışı ani ve hızlı değişirken, diğer kısımlarda ise yavaş ve düzenli değişmektedir. $\mathrm{Bu}$ değişim davranışları singüler pertürbe problemlerin çözümünde sınırsız türevler oluşturur. Uygulanan bazı klasik nümerik metotlar bu sorunları gideremez. Bu nedenle bu çalışmadaki gibi $\varepsilon$ 'a göre kararlılık ve yakınsaklık veren uygun nümerik metotlar tercih edilmelidir. Örneğin sonlu fark metodu, sonlu elemanlar metodu, pertürbasyon teknikleri vb. [1-18]. Singüler pertürbe problemlerin sayısal çözümü ve kararlılık analizi birçok araştırmacı tarafindan 1900'lü yıllardan başlayarak şimdiye kadar yoğun olarak araştırılmışırı [1-18].

$\mathrm{Bu}$ problemler, kimyasal-reaktör teorisi, kontrol teorisi, oşinografi, akışkanlar mekaniği, kuantum mekaniği, hidro mekanik problemler, elektrik ağları ve diğer çeşitli pratik mühendislik sistemlerinde, fen bilimlerinde, tip biliminde, aerodinamik, manyetik dinamik, yayma teori, reaksiyon difüzyon, ışık yayan dalgalar, plazmadaki elektron plazma dalgaları, iletişim hatları, plazma dinamik, arıtılmış gaz dinamik, kütlenin hareketi, plastik, meteoroloji, elektrik akımı, iyon akustik dalgaları ve

"Sorumlu yazar: ayredlanu@gmail.com

Geliş Tarihi: 20.12.2019, Kabul Tarihi: 04.02.2020 
bazı fiziksel modellendirmelerde yer almaktadır [12-14]. Ayrıca bazı araştırmacılar lokal olmayan sınır koşullu singüler pertürbe problemlere de odaklanmışlardır [1-12].

Nümerik integral metodu ise $[15,17,18]$ çalışmalarında Taylor seri açılımı yapıldıktan sonra tüm denklemin integrali alınarak işlemlere devam edilir. Bunlardan farklı olarak [16] çalışmasında ilk olarak verilen denklemin integrali alınır daha sonra sırasıyla fark türevleri kullanılır ve yamuk metodu ile devam edilir. Nihayetinde Thomas algoritması ile yaklaşık çözüm bulunur. Nümerik integral metodu gecikmeli diferansiyel denklemlere [20] ve gecikmeli singüler pertürbe multi-point sınır koşullu problemlere de [21] kolaylıkla uygulanabilir.

Biz de bu çalışmada, [16] makalesinin verdiği motivasyon ile lokal (iki noktalı) sınır şartlı singüler pertürbe problem yerine singüler pertürbe multi-point sınır değer problemlerini nümerik integral metodu ile çözmeyi hedeflemekteyiz. Bu problemi aşağıdaki şekilde verebiliriz:

$$
\begin{aligned}
& \varepsilon u^{\prime \prime}(x)+a(x) u^{\prime}(x)+b(x) u(x)=0,0 \leq x \leq 1, \\
& u(0)=A, u(1)=\sum_{i=1}^{m-2} c_{i} u\left(s_{i}\right)+B
\end{aligned}
$$

$A, B$ ve c c sonlu sabitler; $a(x), b(x)$ ve $f(x)$ sürekli fonksiyonlar; $0<s_{1}<s_{2}<\ldots<s_{m-2}<1$.

Bu problemin katkısı şu şekilde özetlenebilir: Şimdiye kadar, çok noktalı sınır durumlu singüler pertürbe problemler farklı yöntemlerle çözülmüştür. $\mathrm{Bu}$ yöntemlerden farklı olarak singüler pertürbe problemlerin sayısal integral yöntemiyle başarılı bir şekilde çözüldüğü bu çalışmada gösterilmiştir.

Çalışma şu şekilde planlanmıştır: Giriş bölümünde, singüler pertürbe problem ve sayısal integral metodu ile ilgili literatür bilgileri verilir. Bölüm 2' de, sağ (sol) sınır katlı singüler pertürbe problem için sayısal integral metodu ifade edilir. Ayrıca önerilen yöntemin yakınsaklık ve kararlılık analizi de verilir. Bölüm 3' te önerilen yöntemin etkinliğini göstermek için iki test problem incelenir ve sonuçlar Bölüm 4 ' te anlat1lir.

\section{Nümerik İntegral Metodu}

Bu bölümde, çözümü araştırılan singüler pertürbe problem için nümerik integral metodunun işleyiş aşamaları sağ ve sol sınır katları durumlarına göre ayrı ayrı ele alınır. Metodun karalılık şartları verilir.

\subsection{Sağ sınır katlı problem için nümerik integral metodunun çözüm adımları şöyle sıralanır:}

1. (1.1) denkleminde $a(x)<\alpha<0, \alpha$ sabit durumu vardır. Yani $x=1$ sağ sınır katıdır.

2. [0,1] aralığı $h$ uzunluklu olarak $N$ parçaya bölünür. Yani şebeke düzgündür. Buradaki şebeke noktaları ise $0=x_{0}<x_{1} \ldots<x_{n}=1, \mathrm{x}_{i}=x_{0}+i h, \mathrm{i}=0,1, . ., N$ biçimindedir.

3. $i=1, \ldots N-1$ için (1.1) denkleminde her bir terimin $\left[x_{i-1}, x_{i}\right]$ aralığında integrali alınır.

$$
\int_{x_{i-1}}^{x_{i}}\left[\varepsilon u^{\prime \prime}(x)-a(x) u^{\prime}(x)+b(x) u(x)\right] d x=\int_{x_{i-1}}^{x_{i}} f(x) d x, \quad i=1,2, \ldots, N-1,
$$

ve

$$
\varepsilon u^{\prime}\left(x_{i}\right)-\varepsilon u^{\prime}\left(x_{i-1}\right)-a_{i} u\left(x_{i}\right)+a_{i-1} u\left(x_{i-1}\right)+\int_{x_{i-1}}^{x_{i}} b(x) u(x) d x=\int_{x_{i-1}}^{x_{i}} f(x) d x .
$$

4. Adım 3' te yer alan birinci türevler $u^{\prime}\left(x_{i}\right)$ ve $u^{\prime}\left(x_{i-1}\right)$ için $u_{i}^{\prime}=\frac{u_{i+1}-u_{i}}{h}, \quad u_{i-1}^{\prime}=\frac{u_{i}-u_{i-1}}{h}$ fark yaklaşımları kullanılırsa aşağıdaki denklem elde edilir.

$$
\varepsilon\left(\frac{u_{i+1}-u_{i}}{h}\right)-\varepsilon\left(\frac{u_{i}-u_{i-1}}{h}\right)-a_{i} u\left(x_{i}\right)+a_{i-1} u\left(x_{i-1}\right)+\int_{x_{i-1}}^{x_{i}} b(x) u(x) d x=\int_{x_{i-1}}^{x_{i}} f(x) d x .
$$


5. Adım 4' te bulunan integraller için yamuk metodu kullanılır ve

$$
\varepsilon\left(\frac{u_{i+1}-u_{i}}{h}\right)-\varepsilon\left(\frac{u_{i}-u_{i-1}}{h}\right)-a_{i} u_{i}+a_{i-1} u_{i-1}+\frac{h}{2}\left[b_{i} u_{i}+b_{i-1} u_{i-1}\right]=\frac{h}{2}\left[f_{i}+f_{i-1}\right]
$$

denklemi bulunur.

6. Adım 5' te bazı düzenlemeler yapılırsa

7.

$$
u_{i-1}\left(\frac{\varepsilon}{h}+a_{i-1}+h \frac{b_{i-1}}{2}\right)-u_{i}\left(\frac{2 \varepsilon}{h}+a_{i}-h \frac{b_{i}}{2}\right)+u_{i+1}\left(\frac{\varepsilon}{h}\right)=\frac{h}{2}\left[f_{i}+f_{i-1}\right], \quad \mathrm{i}=1,2, \ldots, N-1,
$$

fark denklemi oluşur.

8. Yukarıdaki fark denklemine sınır şartlarını da koyarsak aşağıdaki fark problemi oluşur:

9.

$$
\begin{aligned}
& u_{i-1}\left(\frac{\varepsilon}{h}+a_{i-1}+h \frac{b_{i-1}}{2}\right)-u_{i}\left(\frac{2 \varepsilon}{h}+a_{i}-h \frac{b_{i}}{2}\right)+u_{i+1}\left(\frac{\varepsilon}{h}\right)=\frac{h}{2}\left[f_{i}+f_{i-1}\right], \\
& u_{0}=A, \quad u_{N}=\sum_{i=0}^{m-2} c_{i} u_{N_{i}} .
\end{aligned}
$$

Fark probleminin çözümü aşağıda verilen Thomas algoritması kullanılarak elde edilir.

$$
\begin{gathered}
A_{i}=\frac{\varepsilon}{h}+a_{i-1}+h \frac{b_{i-1}}{2}, \quad \mathrm{~B}_{i}=\frac{\varepsilon}{h}, \quad \mathrm{C}_{i}=\frac{2 \varepsilon}{h}+a_{i}-h \frac{b_{i}}{2}, \quad \mathrm{~F}_{i}=\frac{h}{2}\left[f_{i}+f_{i-1}\right], \\
\alpha_{i+1}=\frac{B_{i}}{C_{i}-\alpha_{i} A_{i}}, \quad \beta_{i+1}=\frac{F_{i}+\beta_{i} A_{i}}{C_{i}-\alpha_{i} A_{i}}, \quad i=1,2, \ldots, N-1, \\
\mathrm{u}_{i}=\alpha_{i+1} u_{i+1}+\beta_{i+1}, \quad i=N-1, \ldots, 2,1,
\end{gathered}
$$

(1.1)-(1.2) problemine göre,

$$
\alpha_{0}=A, \quad \beta_{0}=0
$$

\subsection{Sol sınır katlı problem için nümerik integral metodunun çözüm adımları şöyle sıralanır:}

Sağ sınır katlı problemin çözüm adımlarına benzer olmasına rağmen aşağıdaki adımlar sol sınır katlı probleme $(a(x)>\alpha>0, \alpha$ sabit ) özgüdür. $i=0,1, \ldots N-1$ için (1.1) denkleminde her bir terimin $\left[x_{i+1}, x_{i}\right]$ aralığında integrali alınır.

$$
\varepsilon u^{\prime}\left(x_{i+1}\right)-\varepsilon u^{\prime}\left(x_{i}\right)+a_{i+1} u\left(x_{i+1}\right)-a_{i} u\left(x_{i}\right)+\int_{x_{i}}^{x_{i+1}} b(x) u(x) d x=\int_{x_{i}}^{x_{i+1}} f(x) d x,
$$

Bu denklemde yer alan birinci türevler $u^{\prime}\left(x_{i+1}\right)$ ve $u^{\prime}\left(x_{i}\right)$ için $u_{i+1}^{\prime}=\frac{u_{i+1}-u_{i}}{h}, \quad u_{i}^{\prime}=\frac{u_{i}-u_{i-1}}{h}$ fark yaklaşımları yerlerine yazılır ve aşağıdaki denklem elde edilir. 


$$
\varepsilon\left(\frac{u_{i+1}-u_{i}}{h}\right)-\varepsilon\left(\frac{u_{i}-u_{i-1}}{h}\right)+a_{i+1} u\left(x_{i+1}\right)-a_{i} u\left(x_{i}\right)+\int_{x_{i-1}}^{x_{i}} b(x) u(x) d x=\int_{x_{i-1}}^{x_{i}} f(x) d x,
$$

Yukarıdaki denklemde yer alan integrallere karşılık olarak yamuk metodu kullanılır ve

$$
\varepsilon\left(\frac{u_{i+1}-u_{i}}{h}\right)-\varepsilon\left(\frac{u_{i}-u_{i-1}}{h}\right)+a_{i+1} u_{i+1}-a_{i} u_{i}+\frac{h}{2}\left[b_{i+1} u_{i+1}+b_{i} u_{i}\right]=\frac{h}{2}\left[f_{i+1}+f_{i}\right], \quad i=1,2, \ldots, N-1,
$$

denklemi oluşur.

$$
u_{i-1}\left(\frac{\varepsilon}{h}\right)-u_{i}\left(\frac{2 \varepsilon}{h}+a_{i}-\frac{h b_{i}}{2}\right)+u_{i+1}\left(\frac{\varepsilon}{h}+a_{i+1}+\frac{h b_{i+1}}{2}\right)=\frac{h}{2}\left[f_{i}+f_{i-1}\right], \quad \mathrm{i}=1,2, \ldots, N-1,
$$

fark denklemi oluşur. Bu denkleme sınır şartlarını da eklersek aşağıdaki fark problemi elde edilir:

$$
\begin{aligned}
& u_{i-1}\left(\frac{\varepsilon}{h}\right)-u_{i}\left(\frac{2 \varepsilon}{h}+a_{i}-\frac{h b_{i}}{2}\right)+u_{i+1}\left(\frac{\varepsilon}{h}+a_{i+1}+\frac{h b_{i+1}}{2}\right)=\frac{h}{2}\left[f_{i}+f_{i-1}\right] \\
& u_{0}=A, \quad u_{N}=\sum_{i=0}^{m-2} c_{i} u_{N_{i}}
\end{aligned}
$$

(1.3) fark problemi Thomas algoritması kullanılarak çözülür. Thomas algoritmasına göre,

$$
\begin{gathered}
A_{i}=\frac{\varepsilon}{h}, \quad \mathrm{~B}_{i}=\frac{\varepsilon}{h}+a_{i+1}+\frac{h b_{i+1}}{2}, \quad \mathrm{C}_{i}=\frac{2 \varepsilon}{h}+a_{i}-\frac{h b_{i}}{2}, \quad \mathrm{~F}_{i}=\frac{h}{2}\left[f_{i}+f_{i-1}\right], \\
\alpha_{i+1}=\frac{B_{i}}{C_{i}-\alpha_{i} A_{i}}, \quad \beta_{i+1}=\frac{F_{i}+\beta_{i} A_{i}}{C_{i}-\alpha_{i} A_{i}}, \quad i=1,2, \ldots, N-1, \\
\mathrm{u}_{i}=\alpha_{i+1} u_{i+1}+\beta_{i+1}, \quad i=N-1, \ldots, 2,1,
\end{gathered}
$$

tüm bu adımlar izlenilerek yaklaşık çözüm elde edilir.

\subsection{Kararlılık}

$A_{i}>0, \mathrm{~B}_{i}>0$ ve $\mathrm{C}_{i}>A_{i}+B_{i}>0$,

$\left|\alpha_{i}\right|<1, \quad \mathrm{i}=0,1, \ldots, N-1$,

şartları altında Thomas algoritması kararlıdır. Yine bu şartlar altında fark probleminin çözümü vardır ve tektir [19].

\section{Nümerik İntegral Metodunun Sağ ve Sol Sınır Şartlı Singüler Pertürbe Problemlere Uygulanması}

$\mathrm{Bu}$ bölümde nümerik integral metodunun etkinliğini, kararlılığını ve yakınsaklığını göstermek için iki örnek verilmiştir. İlk örnek sağ sınır katlara, ikinci örnek ise sol sınır katlara sahiptir. 


\section{Örnek 1}

$x=1$ noktasında sağ sınır katı olan singüler pertürbe çok noktalı $\left(x=0, x=\frac{1}{4}, x=\frac{1}{3}, x=\frac{1}{2}, x=1\right)$ problemi nümerik integral metodu ile çözelim.

$$
\begin{aligned}
& -\varepsilon u^{\prime \prime}(x)+u^{\prime}(x)=1,0 \leq x \leq 1, \\
& u(0)=0, u(1)=d+u\left(\frac{1}{4}\right)+2 u\left(\frac{1}{3}\right)+3 u\left(\frac{1}{2}\right), \\
& d \approx-2.41-\frac{6 e^{\frac{-1}{\varepsilon}}}{1-e^{\frac{-1}{\varepsilon}}}+\frac{e^{\frac{-0.75}{\varepsilon}}+2 e^{\frac{-0.66}{\varepsilon}}+3 e^{\frac{-0.5}{\varepsilon}}}{1-e^{\frac{-1}{\varepsilon}}}, \\
& u(x)=x-\frac{e^{\frac{x-1}{\varepsilon}}-e^{\frac{-1}{\varepsilon}}}{1-e^{\frac{-1}{\varepsilon}}},
\end{aligned}
$$

2.1' de sağ sınır katlı problem için verilen nümerik integral metodunun çözüm adımlarını izleyelim:

İlk olarak (1.6) denkleminin her bir terimimin $\left[x_{i-1}, x_{i}\right]$ aralığında integrali alınır ve gerekli düzenlemeler yapılırsa

$$
\begin{gathered}
\int_{x_{i-1}}^{x_{i}}\left[-\varepsilon u^{\prime \prime}(x)+u^{\prime}(x)\right] d x=\int_{x_{i-1}}^{x_{i}} 1 d x, \\
-\varepsilon \int_{x_{i-1}}^{x_{i}} u^{\prime \prime}(x) d x+\int_{x_{i-1}}^{x_{i}} u^{\prime}(x) d x=\int_{x_{i-1}}^{x_{i}} 1 d x, \\
-\varepsilon u^{\prime}\left(x_{i}\right)+\varepsilon u^{\prime}\left(x_{i-1}\right)+u\left(x_{i}\right)-u\left(x_{i-1}\right)=\int_{x_{i-1}}^{x_{i}} 1 d x,
\end{gathered}
$$

elde edilir. Burada,

$u^{\prime}\left(x_{i}\right)$ ve $u^{\prime}\left(x_{i-1}\right)$ için $u_{i}^{\prime}\left(x_{i}\right)=\frac{u_{i+1}-u_{i}}{h}, \quad u_{i-1}^{\prime}\left(x_{i-1}\right)=\frac{u_{i}-u_{i-1}}{h}$ sonlu fark yaklaşımları kullanılır ve aşağıdaki denklem bulunur:

$$
-\varepsilon\left(\frac{u_{i+1}-u_{i}}{h}\right)+\varepsilon\left(\frac{u_{i}-u_{i-1}}{h}\right)+u\left(x_{i}\right)-u\left(x_{i-1}\right)=\frac{h}{2}\left(f_{i}+f_{i-1}\right)
$$

bu denklem $x_{i-1}, x_{i}$ ve $x_{i+1}$ katsayılarına göre yeniden düzenlenir ve

$$
u_{i-1}\left(-\frac{\varepsilon}{h}-1\right)-u_{i}\left(-\frac{2 \varepsilon}{h}-1\right)+u_{i+1}\left(-\frac{\varepsilon}{h}\right)=h,
$$

fark denklemi elde edilir. Bu fark denklemine sınır şartları da eklenirse aşağıdaki fark problemi bulunur: 


$$
\begin{gathered}
u_{i-1}\left(-\frac{\varepsilon}{h}-1\right)-u_{i}\left(-\frac{2 \varepsilon}{h}-1\right)+u_{i+1}\left(-\frac{\varepsilon}{h}\right)=h \\
u\left(x_{0}\right)=u(0)=0, \quad u\left(x_{N}=1\right)-u\left(x_{\frac{N}{4}}\right)-2 u\left(x_{\frac{N}{3}}\right)-3 u\left(x_{\frac{N}{2}}\right)=2 .
\end{gathered}
$$

Artık Thomas algoritması ile $x_{i}$ şebeke noktalarında $u_{i}, i=1,2, \ldots, N-1$ yaklaşık çözümlerine ulaşı1lır.

$$
\begin{gathered}
A_{i}=-\frac{\varepsilon}{h}-1, B_{i}=-\frac{\varepsilon}{h}, \quad C_{i}=-\frac{2 \varepsilon}{h}-1, F_{i}=-h, \\
\alpha_{1}=0, \quad \beta_{1}=0, \\
\alpha_{i+1}=\frac{\left(-\frac{\varepsilon}{h}\right)}{\left(-\frac{2 \varepsilon}{h}-1\right)-\left(-\frac{\varepsilon}{h}-1\right) \alpha_{i}}, \quad \beta_{i+1}=\frac{h+\left(-\frac{\varepsilon}{h}-1\right) \beta_{i}}{\left(-\frac{2 \varepsilon}{h}-1\right)-\left(-\frac{\varepsilon}{h}-1\right) \alpha_{i}}, i=1, \ldots N-1, \\
u_{i}=\alpha_{i+1} u_{i+1}+\beta_{i+1}, i=N-1, \ldots, 2,1 .
\end{gathered}
$$

Thomas algoritmasına göre yukarıda verilen çözüm şeması uygun bir matematik programı kullanılarak uygulanır ve nümerik sonuçlar elde edilir. $N=24,48,96, \ldots 384$ ve $\varepsilon=2^{-2}, 2^{-4}, \ldots, 2^{-38}$ değerleri için maksimum hatalar bulunur. Tam, yaklaşık çözüm ve hata eğrileri bu farklı $N$ ve $\varepsilon$ değerleri için çizilir. Böylece önerilen metodun singüler pertürbe çok noktalı problemler için uygun olduğu gösterilir. Farklı $N$ değerleri ve $\varepsilon$ pertürbasyon parametresi için maksimum mutlak hatalar Tablo 1' de verilir.

Tablo 1. Örnek 1 için Thomas algoritması ile hesaplanan maksimum hatalar

\begin{tabular}{cccccc}
\hline$\varepsilon / N$ & 24 & 48 & 96 & 192 & 384 \\
\hline $2^{-10}$ & 0.0229007627 & 0.0447761203 & 0.0856909703 & 0.1530668055 & 0.2032438190 \\
$2^{-12}$ & 0.0058252420 & 0.0115830129 & 0.0229007603 & 0.0447761249 & 0.0856909697 \\
$2^{-14}$ & 0.0014627007 & 0.0029211313 & 0.0058252399 & 0.0115830173 & 0.0229007511 \\
$2^{-16}$ & 0.0003660762 & 0.0007318875 & 0.0014626985 & 0.0029211358 & 0.0058252314 \\
$2^{-18}$ & 0.0000915324 & 0.0001830965 & 0.0003660741 & 0.0007318917 & 0.0014626899 \\
$2^{-20}$ & 0.0000228867 & 0.0000457989 & 0.0000914943 & 0.0001831732 & 0.0014626899 \\
$\ldots$ & $\ldots$ & $\ldots$ & $\ldots$ & $\ldots$ & $\ldots$ \\
$2^{-35}$ & 0.0000000001 & 0.0000000003 & 0.000000004 & 0.0000000008 & 0.0000000018
\end{tabular}




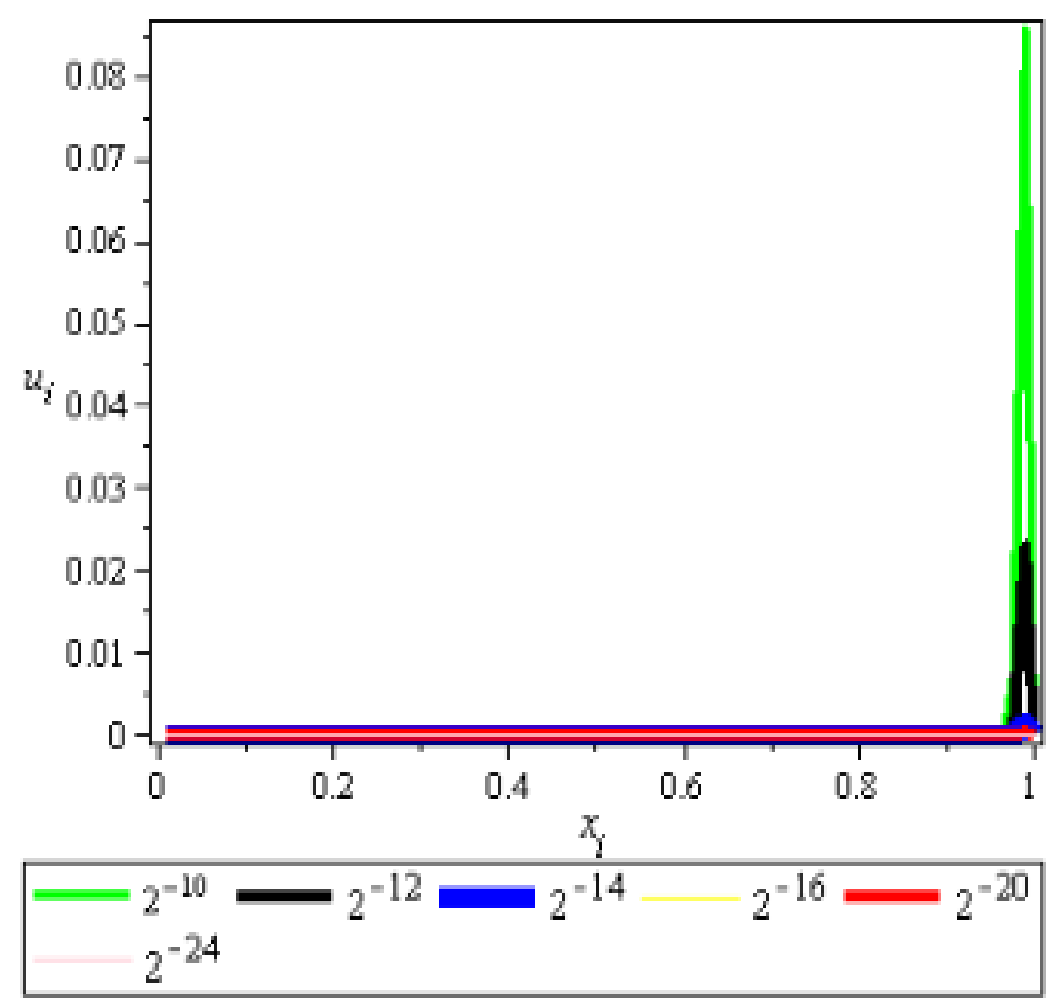

Şekil 1. $N=96$ için hata değerlendirmesi

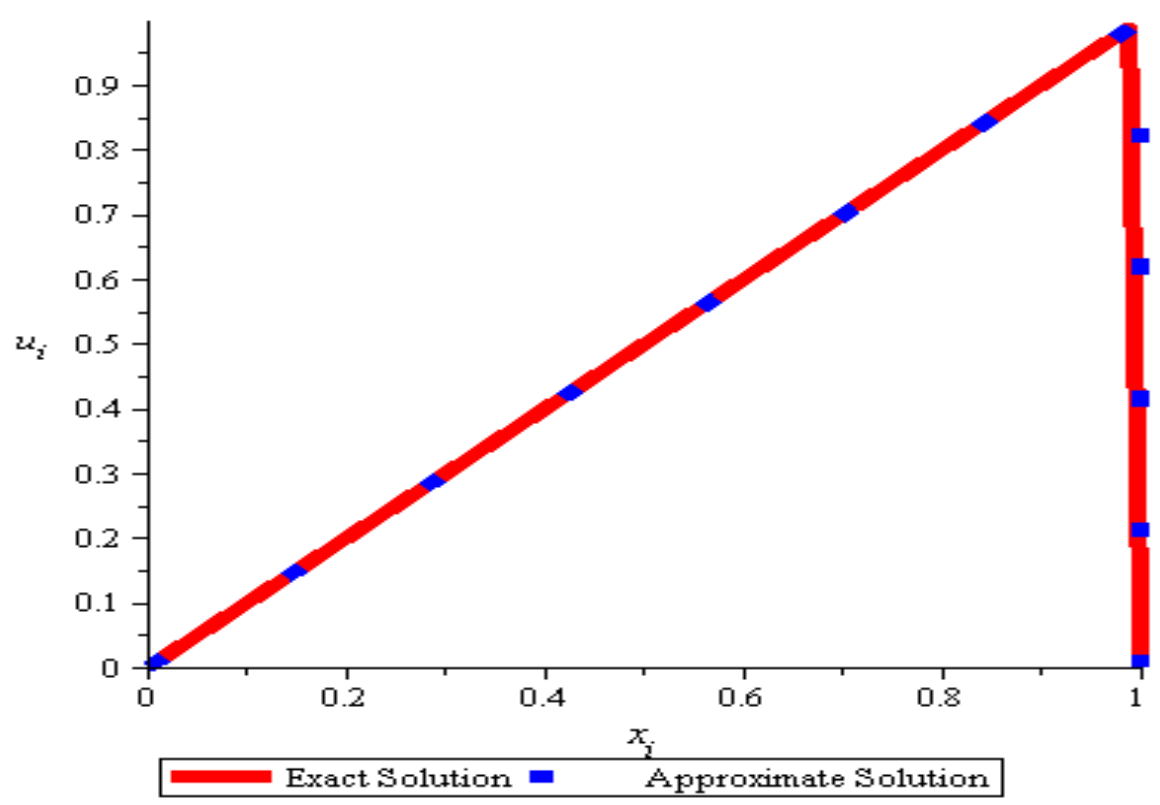

Şekil 2. $N=96, \varepsilon=2^{-20}$ için tam ve yaklaşık çözüm eğrilerinin karşılaştırılması 


\section{Örnek 2}

Şimdi de $x=0$ noktasında sol sınır katına sahip aşağıda verilen çok noktalı $\left(x=0, x=\frac{1}{2}, x=1\right)$ singüler pertürbe problemi nümerik integral metodu ile çözelim.

$$
\begin{aligned}
\varepsilon u^{\prime \prime}(x)+u^{\prime}(x)=1+2 x, \quad 0<x<1, & \\
u(0)=0, \quad \mathrm{u}(1)-u\left(\frac{1}{2}\right)=d, & \\
d & =\frac{1}{4}+\varepsilon-(2 \varepsilon-1) \frac{1-e^{-\frac{1}{2 \varepsilon}}}{1-e^{-\frac{1}{\varepsilon}}}, \\
y(x) & =x(x+1-2 \varepsilon)+(2 \varepsilon-1) \frac{1-e^{-\frac{x}{2 \varepsilon}}}{1-e^{-\frac{1}{\varepsilon}}},
\end{aligned}
$$

(1.7) sol sınır katlı problemde ilk denklemin $\left[x_{i+1}, x_{i}\right]$ aralığında integrali alınır. 2.2' de verilen sol sınır katı çözüm adımları aşağıdaki gibi uygulanırsa:

$$
\begin{gathered}
\int_{x_{i}}^{x_{i+1}}\left[\varepsilon u^{\prime \prime}(x)+u^{\prime}(x)\right] d x=\int_{x_{i}}^{x_{i+1}}(1+2 x) d x \\
\varepsilon \int_{x_{i}}^{x_{i+1}} u^{\prime \prime}(x) d x+\int_{x_{i}}^{x_{i+1}} u^{\prime}(x) d x=\int_{x_{i}}^{x_{i+1}}(1+2 x) d x \\
\varepsilon u^{\prime}\left(x_{i+1}\right)-\varepsilon u^{\prime}\left(x_{i}\right)+u\left(x_{i+1}\right)-u\left(x_{i}\right)=\int_{x_{i}}^{x_{i+1}}(1+2 x) d x, \\
\varepsilon\left(\frac{u_{i+1}-u_{i}}{h}\right)-\varepsilon\left(\frac{u_{i}-u_{i-1}}{h}\right)+u\left(x_{i+1}\right)-u\left(x_{i}\right)=\frac{h}{2}\left(f_{i+1}+f_{i}\right), f_{i}=1+2 x_{i},
\end{gathered}
$$

elde edilir. Buradaki son denklem $x_{i-1}, x_{i}$ ve $x_{i+1}$ katsayılarına göre düzenlenir ve

$$
u_{i-1}\left(\frac{\varepsilon}{h}\right)-u_{i}\left(\frac{2 \varepsilon}{h}+1\right)+u_{i+1}\left(\frac{\varepsilon}{h}+1\right)=h\left(x_{i+1}+x_{i}+1\right) \text {, }
$$

fark denklemi elde edilir. Bu fark denklemi sınır şartları ile birlikte yazılırsa

$$
\begin{aligned}
& u_{i-1}\left(\frac{\varepsilon}{h}\right)-u_{i}\left(\frac{2 \varepsilon}{h}+1\right)+u_{i+1}\left(\frac{\varepsilon}{h}+1\right)=h\left(x_{i+1}+x_{i}+1\right), \\
& u\left(x_{0}\right)=u(0)=0, \quad u\left(x_{N}\right)-u\left(x_{\frac{N}{2}}\right)=d,
\end{aligned}
$$

fark problemi elde edilir. Bu fark problemini çözmek için kullanılan Thomas algoritması aşağıdaki gibi verilir:

$$
A_{i}=\frac{\varepsilon}{h}, \quad B_{i}=\frac{\varepsilon}{h}+1, \quad C_{i}=\frac{2 \varepsilon}{h}+1, \quad F_{i}=-h\left(x_{i+1}+x_{i}+1\right),
$$




$$
\begin{gathered}
\alpha_{1}=0, \quad \beta_{1}=0, \\
\alpha_{i+1}=\frac{\left(\frac{\varepsilon}{h}+1\right)}{\left(\frac{2 \varepsilon}{h}+1\right)-\left(\frac{\varepsilon}{h}\right) \alpha_{i}}, \quad \beta_{i+1}=\frac{h\left(x_{i}+x_{i+1}\right)+\left(\frac{\varepsilon}{h}\right) \beta_{i}}{\left(\frac{2 \varepsilon}{h}+1\right)-\left(\frac{\varepsilon}{h}\right) \alpha_{i}}, i=1, \ldots N-1, \\
u_{i}=\alpha_{i+1} u_{i+1}+\beta_{i+1}, i=N-1, \ldots, 2,1 .
\end{gathered}
$$

Tablo 2' de artan $N$ değerleri ve azalan $\varepsilon$ değerleri için maksimum mutlak hatalar verilir.

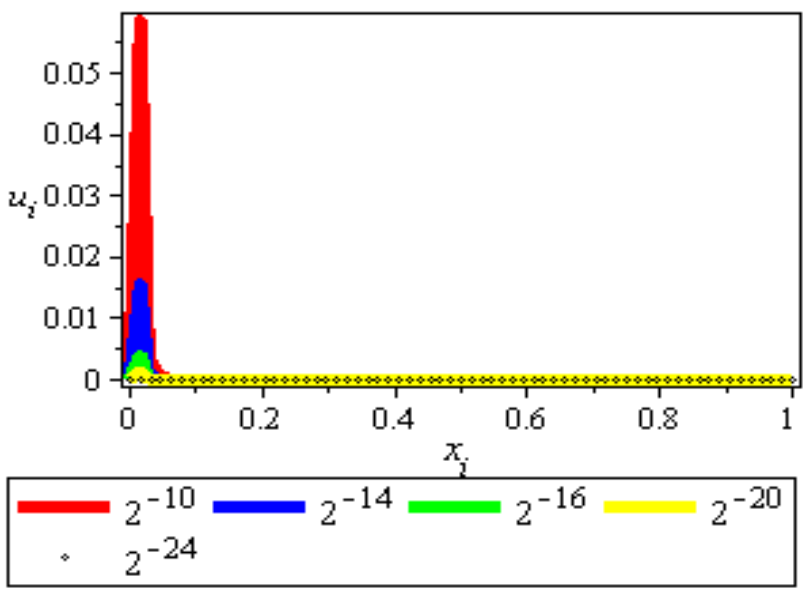

Şekil 3. $N=64$ için hata dağılımı

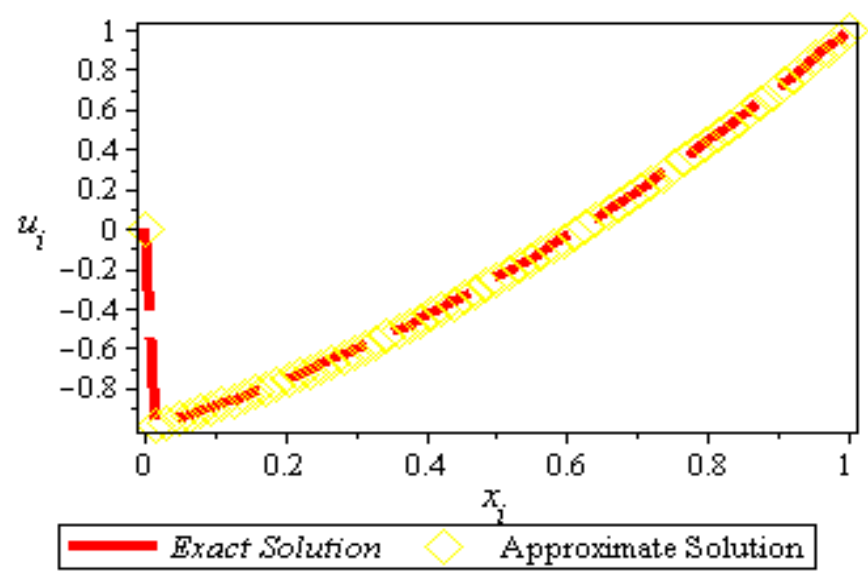

Şekil 4. $N=64, \varepsilon=2^{-20}$ için tam ve yaklaşık çözüm eğrilerinin karşılaştırılması

Tablo 2. Örnek 2 için Thomas algoritması ile hesaplanan maksimum hatalar

\begin{tabular}{cccccc}
\hline$\varepsilon / N$ & 16 & 32 & 64 & 128 & 256 \\
\hline $2^{-10}$ & 0.0153545667 & 0.0302438457 & 0.0587085267 & 0.1105592894 & 0.1813295077 \\
$2^{-12}$ & 0.0038891510 & 0.0077481537 & 0.0153771031 & 0.0302882346 & 0.0587946935 \\
$2^{-14}$ & 0.0009754902 & 0.0019490796 & 0.0038905760 & 0.0077509918 & 0.0153827368 \\
$2^{-16}$ & 0.0002440724 & 0.0004880281 & 0.0009755872 & 0.0019492576 & 0.0038909322 \\
$2^{-18}$ & 0.0000610308 & 0.0001220595 & 0.0002440706 & 0.0004880383 & 0.0009756416 \\
$2^{-20}$ & 0.0000152588 & 0.0000305131 & 0.0000610314 & 0.0001220755 & 0.0002440396 \\
$\ldots$ & $\ldots$ & $\ldots$ & $\ldots$ & $\ldots$ & $\ldots$ \\
$2^{-38}$ & 0.0000000000 & 0.0000000001 & 0.0000000002 & 0.0000000002 & 0.0000000012 \\
\hline
\end{tabular}




\section{Sonuç ve Öneriler}

Sağ ve sol sınır katları içeren singüler pertürbe multi-point (çok noktalı) sınır koşullu problemler nümerik integral metodu ile analiz edilmiştir. [2] çalışmasında ise iki noktalı sınır değer problemleri nümerik integral metodu ile ele alınmıştır. Bizim çalışmamızda çok noktalı sınır değerler ele alındığında nümerik çözüm ile tam çözüm sonuçlarının birbirlerine oldukça yakın olduğu hem Tablo 1 ve 2' de görülen hata değerlerinde hem de tam ve yaklaşık çözüm eğrilerinde görülmektedir. Çünkü tam ve yaklaşık çözüm eğrileri, Şekil 2 ve 4 ' te gösterildiği gibi hemen hemen aynıdır. Şekil 1 ve 3 ' te görüldüğü gibi sınır katı bölgelerinde (Örnek 2 için $x=0$ ve Örnek 1 için $x=1$ ) hatalar, çözümün ani ve hızlı değişiminden dolayı maksimumdur. Önerilen metot her iki sınır katları durumunda da düzgün şebeke üzerinde birinci mertebeden yakınsaktır. Bu sonuçlara göre sayısal veriler ve tüm şekiller, önerilen metodun çok iyi çalıştığını göstermektedir.

\section{Yazarların Katkısı}

Makalede tüm katkı şahsıma aittir.

\section{Çıkar Çatışması Beyanı}

Yazarlar arasında herhangi bir çıkar çatışması bulunmamaktadır.

\section{Araştırma ve Yayın Etiği Beyanı}

Yapılan çalışmada, araştırma ve yayın etiğine uyulmuştur.

\section{Kaynaklar}

[1] Amiraliyev G.M., Cakir M. 2002. Numerical Solution of the Singularly Perturbed Problem with Nonlocal Condition. Applied Mathematics and Mechanics, 23 (7): 755-764.

[2] Cakir M., Arslan D. 2016. A Numerical Method for Nonlinear Singularly Perturbed Multi-Point Boundary Value Problem. Journal of Applied Mathematics and Phyics, 4: 1143-1156.

[3] Cakir M., Arslan D. 2016. Numerical Solution of the Nonlocal Singularly Perturbed Problem. International Journal of Modern Research in Engineering and Technology, 1 (5): 13-24.

[4] Cakir M., Arslan D. 2016. Finite Difference Method for Nonlocal Singularly Perturbed Problem. International Journal of Modern Research in Engineering and Technology, 1 (5): 25-39.

[5] Arslan D. 2017. Finite Difference Method for Solving Singularly Perturbed Multi-Point Boundary Value Problem. Journal of the Institute of Natural and Applied Sciences, 22 (2): 64-75.

[6] Cakir M., Amiraliyev G.M. 2007. Numerical Solution of the Singularly Perturbed Three-Point Boundary Value Problem. International Journal of Computer Mathematics, 84 (10): 1465-1481.

[7] Chegis R. 1988. The Numerical Solution of Problems with Small Parameter at Higher Derivatives and Nonlocal Conditions. Lietuvas Matematica Rinkinys, (in Russian), 28: 144-152.

[8] Arslan D. 2019. Stability and Convergence Analysis on Shishkin Mesh for a Nonlinear Singularly Perturbed Problem with Three-Point Boundary Condition. Quaestiones Mathematicae, 1-14. Doi: 16073606.2019.1636894.

[9] Arslan D. 2019. A Numerical Solution Study on Singularly Perturbed Convection-Diffusion Nonlocal Boundary Value Problem. Communications Faculty of Sciences University of Ankara Series A1 Mathematics and Statistics, 68 (2): 1482-1491.

[10] Gupta C.P., Trofimchuk S.I. 1997. A Sharper Condition for the Solvability of a Three-Point Second Order Boundary Value Problem. Journal of Mathematical Analysis and Applications, 205: 586-597.

[11] Herceg D., Surla K. 1991. Solving a Nonlocal Singularly Perturbed Nonlocal Problem by Splines in Tension. Univ. u Novom Sadu Zb. Rad. Prirod.-Mat. Fak. Ser. Math., 21 (2): 119- 132.

[12] Farell P.A., Miller J.J.H., O.Riordan E., Shishkin G.I. 1996. A Uniformly Convergent Finite Difference Scheme for a Singularly Perturbed Semilinear Equation, SIAM Journal on Numerical Analysis, 33: 1135-1149. 
[13] Miller J.J.H.,, O.Riordan E., Shishkin G.I. 1996. Fitted Numerical Methods for Singular Perturbation Problems. World Scientic, Singapore.

[14] Nayfeh A.H. 1993. Introduction to Perturbation Techniques. Wiley, New York.

[15] Reddy Y.N. 1990. A Numerical Integration Method for Solving Singular Perturbation Problems. Applied Mathematics and Computation, 37: 83-95.

[16] Andargie A., Reddy Y.N. 2008. Numerical Integration Method for Singular Perturbation Problems with Mixed Boundary Conditions. J. Appl. Math. \& Informatics, 26 (5-6): 1273-1287.

[17] Soujanya G., Phnaeendra K. 2015. Numerical Intergration Method for Singular-Singularly Perturbed Two- Point Boundary Value Problems. Procedia Engineering, 127: 545-552.

[18] Ranjan R., Prasad H.S. 2018. An Efficient Method of Numerical Integration for a Class of Singularly Perturbed Two Point Boundary Value Problems. Wseas Transactions on Mathematics, 17: 265-273.

[19] Amirali G., Amirali I. 2018. Nümerik Analiz Teori ve Uygulamalarla. Seçkin Yayıncılık, Ankara, Türkiye.

[20] Altun Y. Tunç C. 2019. New Results on the Exponential Stability of Solutions of Periodic Nonlinear Neutral Differential Systems. Dynamic Systems and Applications, 28: 303-316.

[21] Cimen E. 2017. A Priori Estimates for Solution of Singularly Perturbed Boundary Value Problem with Delay in Convection Term. J. Math. Anal., 8 (1): 202-211. 